\title{
Digital gene expression analysis with sample multiplexing and PCR duplicate detection: A straightforward protocol
}

\author{
Andrey Rozenberg1, Florian Leese ${ }^{2}$, Linda C. Weiss ${ }^{3}$, and Ralph Tollrian¹ \\ ${ }^{1}$ Department of Animal Ecology, Evolution and Biodiversity, Ruhr University Bochum, Bochum, Germany, \\ ${ }^{2}$ Faculty of Biology, Aquatic Ecosystem Research, University of Duisburg and Essen, Essen, Germany, \\ and ${ }^{3}$ Environmental Genomics Group, School of Biosciences, University of Birmingham, Birmingham, UK
}

BioTechniques 61:26-32 (July 2016) doi 10.2144/000114434

Keywords: Tag-Seq; digital expression analysis; SNP discovery; RNA library preparation; PCR duplicates; Daphnia; inducible defenses

Supplementary material for this article is available at www.BioTechniques.com/article/114434.

Tag-Seq is a high-throughput approach used for discovering SNPs and characterizing gene expression. In comparison to RNA-Seq, Tag-Seq eases data processing and allows detection of rare mRNA species using only one tag per transcript molecule. However, reduced library complexity raises the issue of PCR duplicates, which distort gene expression levels. Here we present a novel Tag-Seq protocol that uses the least biased methods for RNA library preparation combined with a novel approach for joint PCR template and sample labeling. In our protocol, input RNA is fragmented by hydrolysis, and poly(A)-bearing RNAs are selected and directly ligated to mixed DNA-RNA P5 adapters. The P5 adapters contain i5 barcodes composed of samplespecific (moderately) degenerate base regions (mDBRs), which later allow detection of PCR duplicates. The P7 adapter is attached via reverse transcription with individual i7 barcodes added during the amplification step. The resulting libraries can be sequenced on an Illumina sequencer. After sample demultiplexing and PCR duplicate removal with a free software tool we designed, the data are ready for downstream analysis. Our protocol was tested on RNA samples from predator-induced and control Daphnia microcrustaceans.

Transcriptome-wide gene expression analysis is commonly performed with the aid of RNA-Seq (1). With high enough coverage, it is possible to reconstruct the transcriptome under investigation de novo $(2,3)$. Currently, RNA-Seq is mainly used for assessing gene expression. RNA-Seq data analysis is complicated by rather sophisticated bias correction and normalization procedures (3-5). Tag-Seq, a class of digital gene expression (DGE) methods, approaches the same task by delivering only one fragment for each transcript; this avoids many of the computational issues associated with RNA-Seq, and the resulting sequence tags can be directly used as proxies for gene expression levels. In addition, as tag counts are not dependent on gene length, Tag-Seq is more powerful for differential gene expression analysis $(6,7)$.

The Tag-Seq method of choice has been a modification of serial analysis of gene expression (SAGE) (8), in which transcript tags are prepared with the aid of restriction enzymes (6,7,9-11). The length of the tags does not exceed 27 bases $(7,10)$, thus imposing narrow limits on the mappability of the resulting reads. Moreover, such libraries have inherently low complexity, and duplicates arising during the indispensable PCR step may become a major obstacle in assessing expression levels when the number of PCR cycles is moderately high (10), and/or when sequencing is deep.

Some restriction-free Tag-Seq protocols address these shortcomings by utilizing random priming: short primers tailed with sequencing adapters anneal to cDNA at random positions to produce fragments of suitable length $(12,13)$ (Application Note: Moll et al. QuantSeq 3' mRNA sequencing for RNA quantification. Nat. Methods 11:i-i.). The tag length is less restricted, and PCR duplicates are limited due to differences in the starting positions of the reads. Analogously, a higher library complexity can be achieved by random shearing. Thus, in CEL-Seq (14), RNA is ligated to adapters and sheared with subsequent selection of adapter-bearing 3 ' ends. A similar approach is utilized in the proprietary MACE technology (15-17), in which shearing is performed on cDNA molecules bound to magnetic

\section{METHOD SUMMARY}

We have developed a new protocol for digital expression profiling that is designed to produce single sequencing tags close to the 3 ' ends of transcripts. The main advantages of our approach are strand specificity, reduced bias during library preparation, and detection of PCR duplicates using modified barcodes composed of degenerate sequences for joint sample and PCR-template tagging. 

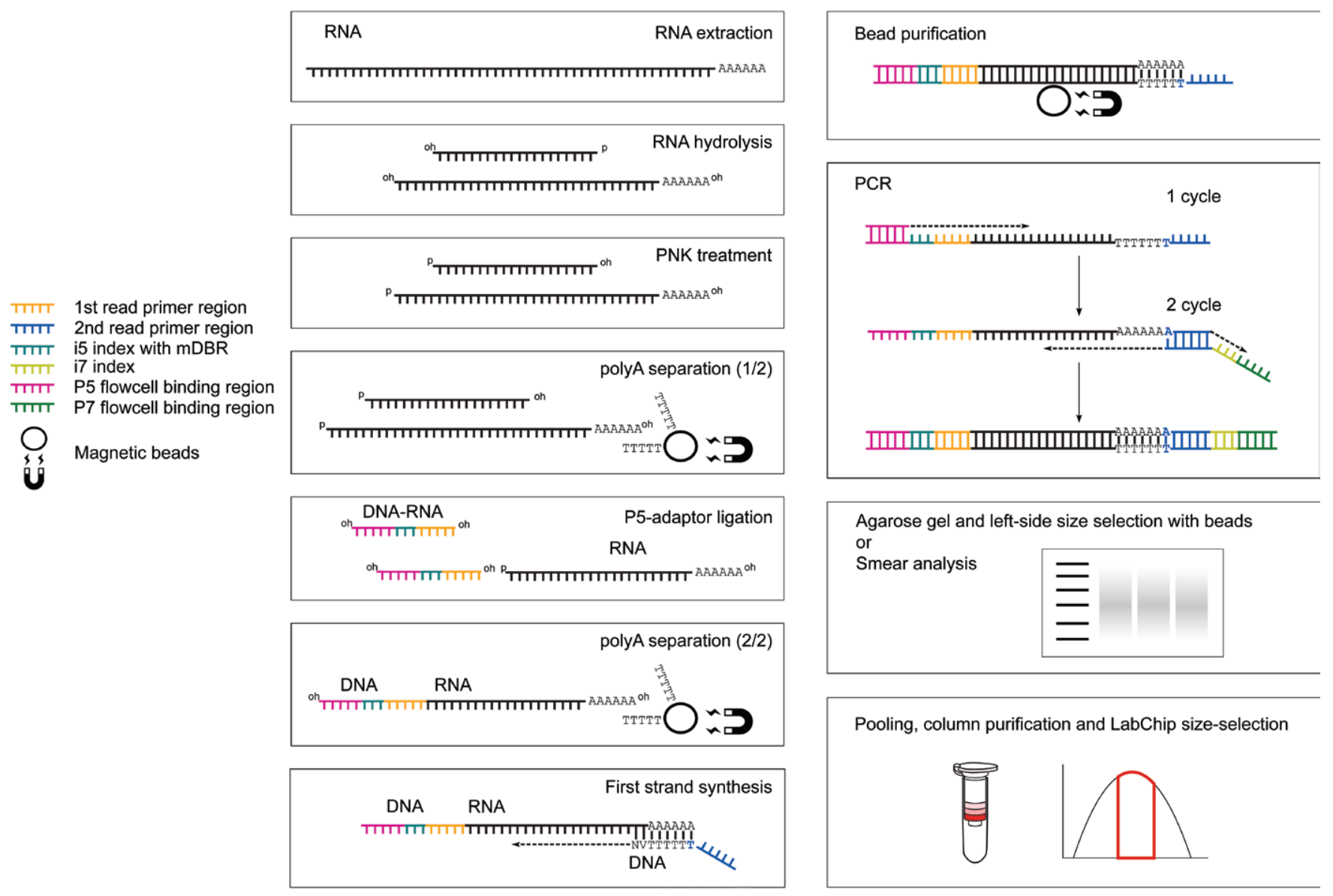

Agarose gel and left-side size selection with beads

or

Smear analysis

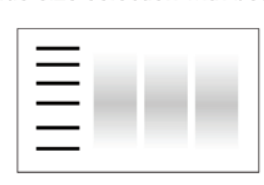

Pooling, column purification and LabChip size-selection

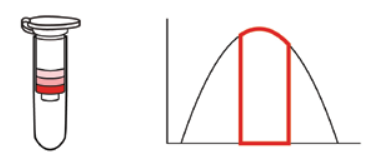

Figure 1. Laboratory workflow. Outline of the proposed Tag-Seq protocol with modifications to the nucleic acids. Sequence regions relevant for sequencing on Illumina machines are color-coded.

beads. Notwithstanding the higher library complexity in these Tag-Seq methods, the range of the potential read starting positions is relatively narrow, which hinders PCR duplicate detection for genes with read counts above a certain limit.

Other approaches to avoid PCR duplication, by performing amplification on microbeads or avoiding it altogether, have also been proposed (18-20), but these have not been further developed, presumably because of short read lengths and the relative unpopularity of the respective sequencing instruments.

Here, we introduce a simple and straightforward protocol for Tag-Seq optimized for Illumina sequencing platforms. This method avoids the problems associated with other approaches and provides a robust way to discern PCR duplicates. In addition to the template labeling principle suggested earlier (21-23), our protocol produces libraries with individual PCR templates labeled with (moderately) degenerate base regions (mDBRs). The mDBR indices are simultaneously used for sample tagging, which provides an additional dimension for multiplexing.
We tested the new protocol by analyzing the transcriptional basis of the difference between defended and undefended morphs of the clonal microcrustacean Daphnia pulex. In the presence of its predator, the phantom midge larvae Chaoborus, D. pulex develop tiny protective neckteeth during early juvenile instars (24). The neckteeth are induced by predator-specific chemicals known as kairomones. Such defenses are only employed when needed, so associated costs are saved in the absence of the predator $(25,26)$.

\section{Materials and methods}

Materials

Six females of North American Daphnia pulex clone $\mathrm{R} 9$ were individually placed in glass vials $(50 \mathrm{~mL})$ when embryos in their brood pouches had developed to early stage IV. Half of the vials contained a 20\%-dilution of water exposed to 50 Chaoborus larvae fed on 500 juvenile daphniids in a $1 \mathrm{~L}$ jar (the kairomone treatment). The other half contained water without predator cues (the control). When the juveniles left the mothers' pouches, the adults were removed, and the medium was changed. Thirty hours after induction, the juveniles were fixed in RNAlater (Qiagen, The Netherlands). Libraries were prepared for each of the six samples corresponding to the individual clutches, and two samples were further split into two after RNA extraction to provide technical replicates (Table 1).

As an additional verification of the protocol and to test its applicability for degraded material, a set of Tag-Seq libraries was prepared for 8 other RNAlaterfixed Daphnia samples and 11 samples of alcohol-fixed mayfly larvae (Insecta: Ephemeroptera).

\section{Library preparation protocol}

The general workflow is outlined in Figure 1 and a detailed manual can be found in the Supplementary Material. The library preparation steps take 2 days. The procedures up until cDNA synthesis are best performed on the same day.

\section{RNA extraction}

Total RNA can be extracted with any suitable kit for the respective tissue type; 
Table 1. Daphnia experimental samples and the respective Tag-Seq libraries. The two samples for which technical replicates were prepared are additionally labeled with numbers in parentheses.

\begin{tabular}{|c|c|c|c|c|c|}
\hline Sample & Treatment & Library & P7 primer & P5 adapter & ExFold mix \\
\hline jl30c2 (1) & Control & jl30c2A & ¡705 & P5ND1A & 2 \\
\hline jl30c2 (2) & Control & jl30c2B & i706 & P5ND1 & 1 \\
\hline j130c3 & Control & jl30c3 & i707 & P5ND1 & 2 \\
\hline j130c4 & Control & jl30c4 & $i 708$ & P5ND3 & 1 \\
\hline j130n 1 & Kairomone & j130n1 & i709 & P5ND1 & 2 \\
\hline j130n2 & Kairomone & jl30n2 & $i 710$ & P5ND4 & 1 \\
\hline jl30n4 (1) & Kairomone & jl30n4A & i711 & P5ND1A & 2 \\
\hline jl30n4 (2) & Kairomone & jl30n4B & i712 & P5ND1 & 1 \\
\hline
\end{tabular}

we used the RNeasy Micro Kit (Qiagen, Venlo, the Netherlands) following the manufacturer's protocol, but omitting the DNase treatment step. (The mayfly RNA was co-extracted with Qiagen's DNeasy Blood and Tissue Kit.) After extraction, RNA quality and quantity were controlled; we employed a Qubit 2.0 fluorometer with the RNA BR Assay Kit (Life Technologies, Carlsbad, CA) and an Experion with the RNA StdSens Analysis Kit (Bio-Rad, Berkeley, CA). Each sample (100-300 ng) was diluted in $16 \mu \mathrm{L}$ nuclease-free water for down-stream analysis.

RNA fragmentation and PNK treatment If required, spike-in controls must be added before this step. For the test run, we added a small amount of ERCC ExFold RNA Spike-In Mix 1 or 2 (Life Technologies) to each library (see Table 1). RNA was hydrolyzed by adding $4 \mu \mathrm{L}$ 10x Polynucleotide Kinase Buffer [New England BioLabs (NEB), Ipswich, MA] and incubating the mix at $94^{\circ} \mathrm{C}$ for a short period of time. (This may need adjustment; we applied 1:40 min for high-quality RNA and 0-1 min for the degraded material.) The rest of the polynucleotide kinase reaction mix was added afterwards (total volume: $20 \mu \mathrm{L}$ ): $9 \mu \mathrm{L}$ nuclease-free water, $1 \mu \mathrm{L} 40 \mathrm{U} / \mu \mathrm{l}$ Murine RNase Inhibitor (NEB), $4 \mu \mathrm{L} 10 \mathrm{mM}$ ATP (NEB), $4 \mu \mathrm{L} 1 \mathrm{mM}$ spermidine (SigmaAldrich, St. Louis, MO), and $2 \mu \mathrm{L} 10 \mathrm{U} / \mu \mathrm{l}$ T4 Polynucleotide Kinase (NEB).

Poly(A)-fragment isolation

Reaction clean-up and simultaneous isolation of mRNA were performed with oligo $d(T)_{25}$ magnetic beads (NEB) following the manufacturer's recommendations, except that $13 \mu \mathrm{L}$ of the resuspended beads were taken, only 1 round of bead binding was performed, and the elution was carried out with a mix of $10.5 \mu \mathrm{L}$ nucleasefree water, $2.8 \mu \mathrm{L} 10 \mathrm{mM}$ ATP, and $1.4 \mu \mathrm{L}$ 20 4M P5 adapter (library-specific; see Tables 1 and 2). (All oligonucleotides were synthesized by Biomers, Germany.)

\section{P5 adapter ligation}

The eluate was incubated at $65^{\circ} \mathrm{C}$ for 2 min and then placed on ice. Other components of the ligation mix were added (total volume: $28 \mu \mathrm{L}$ ): $2.8 \mu \mathrm{L} 10 \times$ T4 RNA ligase buffer (NEB), $5.6 \mu \mathrm{L} 50 \%$ PEG8000 (NEB), 2.8 L DMSO (SigmaAldrich), $0.7 \mu \mathrm{L}$ RNase Inhibitor, and $1.4 \mu \mathrm{L} 30 \mathrm{U} / \mu \mathrm{l}$ T4 RNA ligase 1 (NEB). The mix was incubated at $25^{\circ} \mathrm{C}$ for 2 hours.

\section{Reverse transcription}

The ligation reaction was purified with the same oligo $d(T)_{25}$ magnetic beads left after the mRNA isolation. It was performed in the same way, but the RNA was eluted with $14.5 \mu \mathrm{L} 6.9 \mu \mathrm{M}$ P7-TnVN reverse transcription primer (Table 2). The eluate was incubated at $65^{\circ} \mathrm{C}$ for 2 min and placed on ice. Other components of the reverse transcription reaction were added (total volume: Synthesis Reaction Buffer (NEB), $0.5 \mu \mathrm{L}$ $20 \mu \mathrm{L}): 4 \mu \mathrm{L} 5 \times$ NEBNext First Strand

Table 2: Oligonucleotides used for library construction.
RNase Inhibitor, and $1 \mu \mathrm{L}$ ProtoScript II Reverse Transcriptase (NEB). The mix was incubated for $10 \mathrm{~min}$ at $25^{\circ} \mathrm{C}, 1 \mathrm{~h}$ at $42^{\circ} \mathrm{C}, 20 \mathrm{~min}$ at $65^{\circ} \mathrm{C}$, and then cooled to $4^{\circ} \mathrm{C}$.

\section{PCR}

The reaction was cleaned-up by adding 1.8× (36 $\mu \mathrm{L})$ resuspended Agencourt AMPure XP magnetic beads (Beckman Coulter, Brea, CA) (see Supplementary Material for details). The cDNA was eluted with the following components of the PCR mix: $12.2 \mu \mathrm{L}$ water, $2.5 \mu \mathrm{L}$ 5 4M P5 primer, $2.5 \mu \mathrm{L} 5 \mu \mathrm{M}$ P7 (Illumina i7) primer (library-specific; see Table 2), and $2.5 \mu \mathrm{L} 2 \mathrm{mM}$ (each) dNTPs (NEB). Other components were added after elution (total volume: $25 \mu \mathrm{L}$ ): $5 \mu \mathrm{L} 5 \times \mathrm{Q} 5$ Polymerase Buffer (NEB) and $0.25 \mu \mathrm{L}$ $2 \mathrm{U} / \mu \mathrm{l}$ Q5 Polymerase (NEB).

The mix was incubated for $30 \mathrm{~s}$ at $98^{\circ} \mathrm{C}$ and then each cycle included $10 \mathrm{~s}$ at $98^{\circ} \mathrm{C}, 30 \mathrm{~s}$ at $65^{\circ} \mathrm{C}$, and $30 \mathrm{~s}$ at $72^{\circ} \mathrm{C}$. The incubation was finalized with an elongation step of 2 min at $72^{\circ} \mathrm{C}$. The number of PCR cycles must be checked empirically. For the test run, we used 12 cycles and visualized the PCR products on gel with the subsequent addition of 2 more cycles for samples with low yield. For the second run, we applied 10 PCR cycles and analyzed the DNA on a Fragment Analyzer (Advanced Analytical, Heidelberg, Germany).

\begin{tabular}{|c|c|c|c|}
\hline Name/Sequence & Step & Nucleotides & Index \\
\hline P7-TnVN primer & Reverse transcription & 49 & \\
\hline \multicolumn{4}{|c|}{ CTGGAGTTCAGACGTGTGCTCTTCCGATCT-TTTTTTTTTTTTTTTTT-VN } \\
\hline P5 primer & PCR & 38 & \\
\hline \multicolumn{4}{|c|}{ AATGATACGGCGACCACCGAGATCTACAC } \\
\hline P5 mDBR 1A (P5ND1A) adapter & Ligation & 73 & NNYRYYRR \\
\hline \multicolumn{4}{|c|}{ AATGATACGGCGACCACCGAGATCTACAC-YYRRYRNN-ACACTCTTTCCCTACACGACGCTCTTCCGATCT-r(NNN } \\
\hline P5 mDBR 1 (P5ND1) adapter & Ligation & 72 & NNYRYYRR \\
\hline \multicolumn{4}{|c|}{ AATGATACGGCGACCACCGAGATCTACAC-YYRRYRNN-ACACTCTTTCCCTACACGACGCTCTTCCGATC-r(UNN) } \\
\hline P5 mDBR 2 (P5ND2) adapter & Ligation & 72 & NNRRYRYY \\
\hline \multicolumn{4}{|c|}{ AATGATACGGCGACCACCGAGATCTACAC-RRYRYYNN-ACACTCTTTCCCTACACGACGCTCTTCCGATC-r(UNN) } \\
\hline P5 mDBR 3 (P5ND3) adapter & Ligation & 72 & NNYYRRYR \\
\hline \multicolumn{4}{|c|}{ AATGATACGGCGACCACCGAGATCTACAC-YRYYRRNN-ACACTCTTTCCCTACACGACGCTCTTCCGATC-r(UNN) } \\
\hline P5 mDBR 4 (P5ND4) adapter & Ligation & 72 & NNRYRYRY \\
\hline \multicolumn{4}{|c|}{ AATGATACGGCGACCACCGAGATCTACAC-RYRYRYNN-ACACTCTTTCCCTACACGACGCTCTTCCGATC-r(UNN) } \\
\hline P7 index 5 (i705) primer & PCR & 66 & ATTCAGAA \\
\hline \multicolumn{4}{|c|}{ (see NEB's “Multiplex Oligos for Illumina”) } \\
\hline P7 index 6 (i706) primer & PCR & 66 & GAATTCGT \\
\hline P7 index 7 (i707) primer & PCR & 66 & AGCTTCAG \\
\hline P7 index 8 (i708) primer & PCR & 66 & GCGCATTA \\
\hline P7 index 9 (i709) primer & PCR & 66 & CATAGCCG \\
\hline P7 index 10 (i710) primer & PCR & 66 & TTCGCGGA \\
\hline P7 index 11 (i711) primer & PCR & 66 & GCGCGAGA \\
\hline P7 index 12 (i712) primer & PCR & 66 & CTATCGCT \\
\hline
\end{tabular}




\section{Quantitation and pooling}

When PCR products are analyzed on a gel, primers must be removed with AMPure XP beads (Beckman Coulter) to obtain accurate measurements of the DNA content. Based on the concentration measurements (we used Qubit 2.0 with dsDNA HS Assay Kit), samples to be sequenced on the same lane were equimolarly pooled. The total amount of DNA in our test pool was $8 \times 78 \mathrm{ng}$. If the PCR products are analyzed with a highresolution instrument (as was the case in our second experiment), the pooling can be performed directly based on the results of the smear analysis. The total amount of DNA in the target size range for the second run was $19 \times 3.15 \mathrm{ng}$.

The pooled samples are columnpurified, for which we utilized a MinElute Reaction Cleanup Kit (Qiagen). The final size-selection step is performed with LabChip or similar device (LabChip XTe with DNA 750 Assay Kit (PerkinElmer, Waltham, MA) was at our disposal) set to select the range of 319-478 nucleotides.

\section{Sequencing}

Any combination of Illumina Sequencer (Illumina Inc., San Diego, CA) and its respective kit capable of sequencing dual-indexed libraries can be used. Read lengths of 50-100 nucleotides can be recommended based on our observations (see "Results and discussion" section). The fragments are read only from one direction but with both indices. For testing, we used the Illumina NextSeq 500 with the NextSeq 500 Mid Output v2 Kit with 150 cycles. Our data are available from the NCBI Sequence Read Archive (accession: SRP072662).

\section{Data processing}

Quality filtering and demultiplexing: Any suitable program for quality filtering can be used; we used trimmomatic v. 0.32 (27) coupled with a custom script for additional filtering and poly(A)-tail trimming (polya trim.pl; https://github.com/har-wradim/ tagseq). The reads were then demultiplexed and PCR duplicates were removed with a tool we designed for this purpose (https://github.com/har-wradim/tagseq) (see Supplementary Material for details).

Data analysis: Demultiplexed reads were mapped to a reference transcriptome or genome and respective differential expression and/or SNP detection analyses were performed with the software of
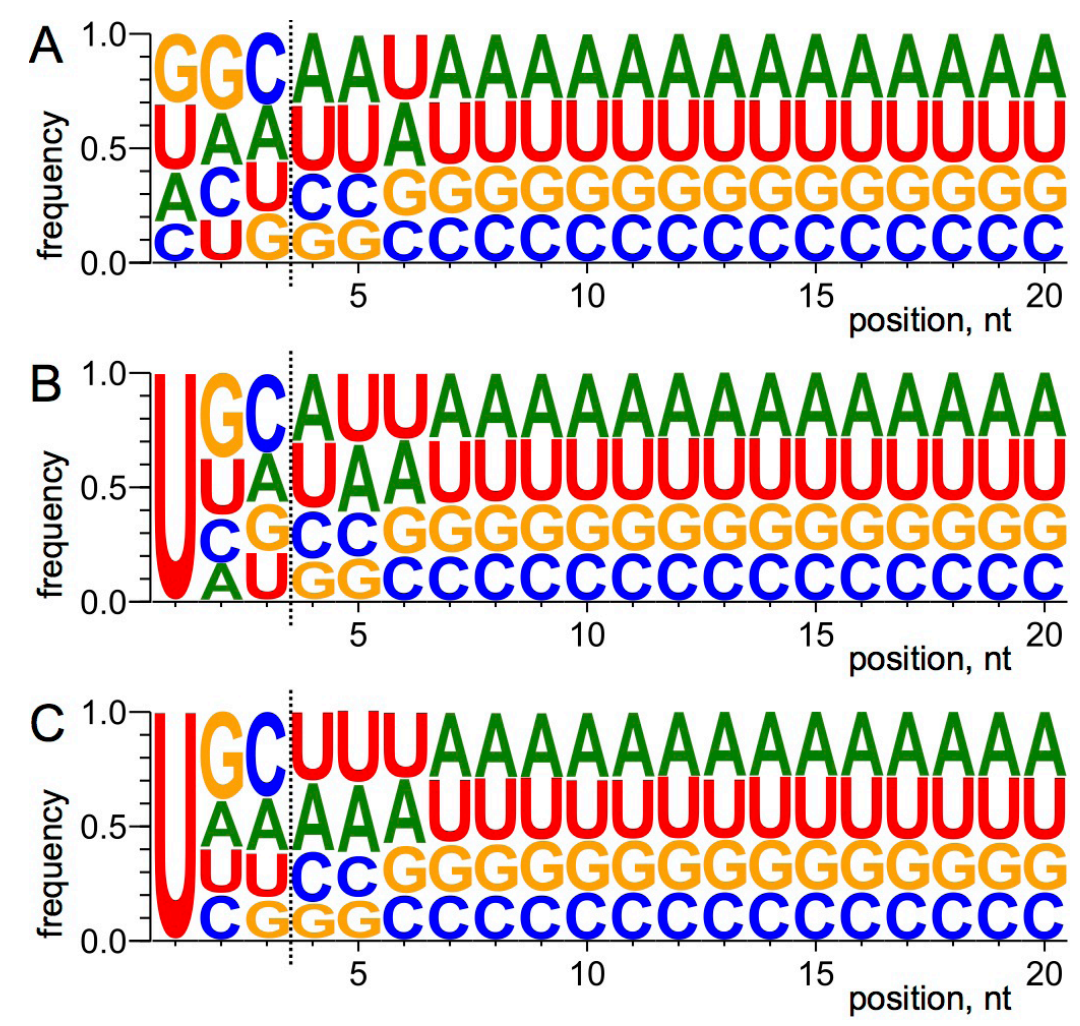

Figure 2. Nucleotide frequencies at the ligation sites of the P5 adapters exemplified by three libraries. (A) j130c2 and adapter P5ND1A (with the r(NNN) tail); (B) jl30c2 and adapter P5ND1 (r(UNN) tail) and (C) jl30n2 and P5ND4 (r(UNN) tail). The vertical dotted line indicates the boundary between the adapter ribotail and the RNA fragment insert; the invariant " $U$ " in (B) and (C) belongs to the non-observed part of the adapter and is added for illustrative purpose. nt: nucleotide.

choice. For the test libraries, we mapped the reads with Bowtie 2 v. 2.2.5 (28). If not stated otherwise, reads were trimmed to 50 nucleotides before mapping. Differential expression analysis was performed with DESeq2 v. 1.6.2 (29). SNP discovery was assisted by samtools and bcftools v. 1.3 (30). The bioinformatic workflows were organized in bash v. 4.3.11 (www.gnu. org/software/bash/) and R v. 3.2.1 (www.rproject.org/) with the help of common *nix commands and $\mathrm{R}$ packages and custom Perl and awk scripts.

\section{Reference transcriptomes}

For an optimal mapping rate of Daphnia reads, we assembled a clone-specific reference transcriptome. For this purpose, 4 RNA-Seq libraries from pools of induced and non-induced juvenile Daphnia pulex R9 were sequenced on an Illumina HiSeq 2000 with a total yield of $158,264,691$ read pairs $(2 \times 100$ nucleotides) and assembled with Trinity r. 20140717 (31). Two other assemblies were performed as well: one based on the Tag-Seq data only and another based on the RNA-Seq and Tag-Seq reads joined together. For
SNP discovery, reads were mapped to the mRNA "Genes 2010" prediction set available for a closely related reference species from http://wfleabase.org.

\section{Results and discussion}

\section{Protocol Design}

Our protocol was designed for moderate amounts of input RNA (100-1000 ng) available from small invertebrates. As demonstrated by the success of our second experiment, the protocol can also be applied to degraded material such as samples subjected to alcohol fixation or RNA co-extracted with DNA, provided that enough RNA fragments of considerable length can be obtained. The laboratory workflow was specifically planned to reduce biases, expense, time, and complexity of the library preparation process. Methods known to have less bias in RNA library preparation were used, while different steps were coupled as much as possible to avoid unnecessary clean-up steps. RNA fragmentation was carried out by heating, which was shown previously to be superior to RNase treatment (32). Direct P5 adapter RNA ligation was performed 

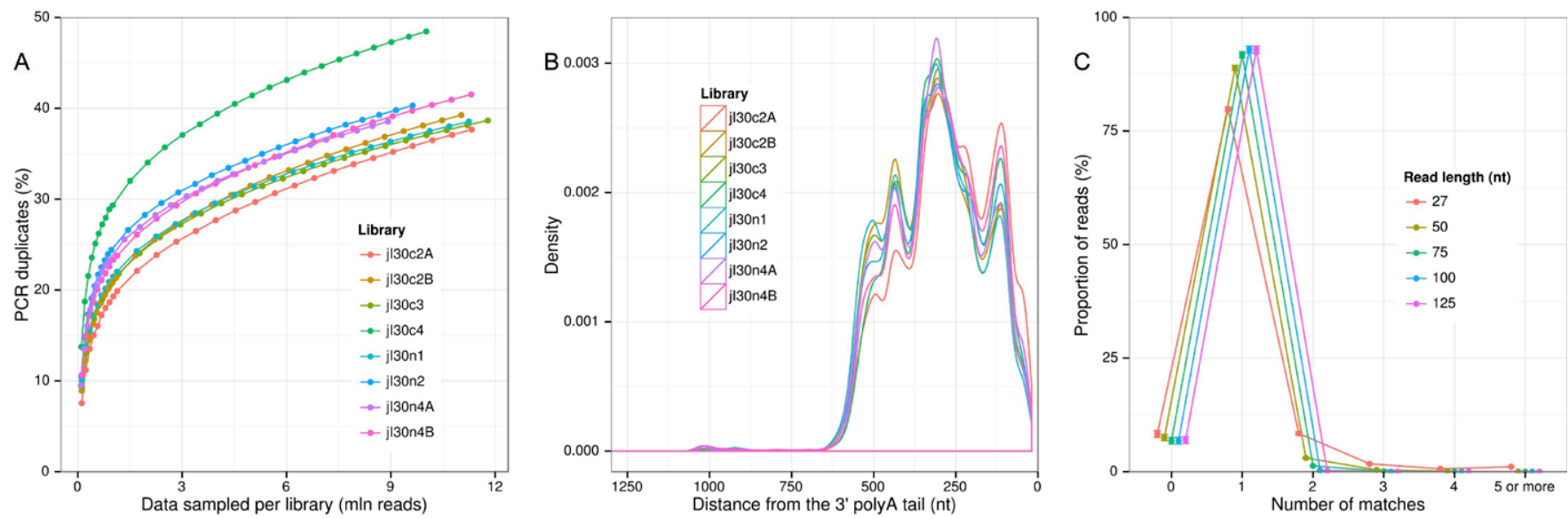

Figure 3. Tag-Seq reads: PCR duplicate abundances, positions relative to the Poly(A) tail, and mappability. (A) Dependence of proportion of PCR duplicates on the number of sampled reads. A total of 12-14 PCR cycles were applied. The whole set of reads was randomly subsampled to produce fractions of the total number of reads. The right-most points correspond to the whole data set. (B) Density distributions for the distances of read starting positions relative to the poly(A) tail. The calculations are based only on the reads mapped to ExFold spike-In RNAs with lengths of at least 1000 excluding the poly(A) tail. (C) Mapping rates for Tag-Seq reads of different lengths against the reference transcriptome assembled from the RNA-Seq data only. Matches were counted for genes, as defined by Trinity r. 20140717.

right after the fragmentation step, which is one of the least biased approaches among RNA library preparation methods (13) and also eliminates the need for a secondstrand synthesis step before PCR. Since only the terminal positions of the adapter sequence are required to be ribobases (33), we designed DNA adapters with a threebase ribotail (see Table 2) instead of the expensive and less stable RNA adapters normally used for direct RNA-RNA ligation $(5,13,34)$. In order to avoid the potential bias of RNA ligase(s) in terms of base composition of the ligated ends $(34,35)$, we included two or three degenerate bases in the ribotail as recommended by Jayaprakash et al. (34) for miRNA sequencing. We did not attempt to disentangle the biases introduced by ligation from those of hydrolysis, but the net result of these two factors on the base composition of the RNA donor at the ligation site is negligible for both ribotails (Figure 2). This enables the use of the shorter adapters (P5ND1-4; see Table 2).

We used Illumina's dual-indexing system not only for samples but also for PCR-template tagging. As in a standard dual-indexing workflow, the P7 and P5 indices (i7 and i5) provide two dimensions for sample labeling. Our modification of i5 consists of a (moderately) degenerate base region (mDBR) to allow the use of individually tagged PCR templates (Table 2). In our scheme, there were four such mDBR indices, each differing in at least three positions. The total number of mutually compatible mDBRs can be increased to eight (see Supplementary Material). One mDBR can tag up to 1024 PCR templates with the same sequence, which is equivalent to 10 bits of
Shannon's entropy if the base frequencies at the variable positions are exactly equal. The observed variability of the i5 sequences in the data we obtained ranged from 9.774 to 9.939 bits, depending on the oligonucleotide preparation. Thanks to their degeneracy, each of the P5 indices is expected to provide enough base variability to avoid the so-called blinding effect. (Supplementary Material for a detailed discussion on the advantages of the proposed coding scheme.)

The P7 indices used are from the New England BioLabs "Multiplex Oligos." Their total number is 12 , thus allowing us to combine $4 \times 12$ or $8 \times 12$ samples on a single sequencing lane. (The number of the i7 indices as well as the index lengths can be further increased to allow for more coding capacity.)

Similar coding principles can be utilized in other low-complexity library preparation methods.

\section{Results of the test run}

Overall, 118,507,435 reads (150 nucleotides long) were obtained from the sequencer. Almost all (99.9\%) of the raw reads were retained after the adapter trimming step, and $78.0 \%$ of all reads were retained after quality trimming. The relatively high number of rejected reads was associated with multiple failed $i 7$ reads. Since this was not observed with the second set of libraries, it must be considered an idiosyncrasy of this particular run. Most (92.3\%) of the 92,371,345 reads that passed the filtering steps could be assigned to the respective libraries.

The rates of detected PCR duplicates per library as a function of the data set size are presented in Figure 3A. (See also Supple- mentary Figure S1 for the results from the second sequencing run.) One of the differences between the runs was that in the first, 12-14 PCR cycles were applied and in the second, only 10 cycles were done. While in most of the RNA- and Tag-Seq experiments the exact number of duplicates remains unknown, with our PCR template tagging, PCR duplicates can be effectively identified, counted, and filtered out. Nevertheless, in order to maximize the yield of the unique reads, it is advisable to keep the number of PCR cycles as low as possible.

Of the 624,036 reads mapped to the spike-in control RNAs, only 3 were in reverse orientation, indicating very high strand specificity. Reads matching the control RNAs with a length of at least 1000 nucleotides were used to analyze the distribution of read starting positions (Figure 3B). The vast majority of the reads started near the $3^{\prime}$ end of the RNA. The discernible peaks likely correspond to the so-called points of fragment accumulation characteristic to the RNA hydrolysis reaction (36).

Reads trimmed to $27,50,75,100$, and 125 nucleotides were mapped against 3 transcriptome assemblies, and the rates of different numbers of matches for genes and isoforms were analyzed (Figures 3C, Supplementary Figure S2). The overall mapping rate for the 50-nucleotide long reads ranged from $92.5 \pm 0.57 \%$ (mean \pm SD) for the RNA-Seq-based assembly to $95.3 \pm 0.14$ for the assembly based on the combined RNA-Seq and Tag-Seq data. The number of the uniquely mapped reads reached saturation between 75 and 100 nucleotides, while 50 nucleotide reads had very similar mapping rates. The short 
27-nucleotide reads matched multiple targets disproportionately more often than longer reads (Supplementary Figure S2). These observations indicate that Tag-Seq reads of lengths in the range of 50-100 nucleotides are sufficient for mapping. As expected, the ability of the Tag-Seq reads to differentiate isoforms of the same genes appeared very limited and was restricted to genes with variable 3' exons (data not shown).

Two biological samples were technically replicated to test for reproducibility (Table 1). The resulting plots of the per-gene expression levels for the pairs of replicates show our method to be very reproducible (Figure 4A). Similar results were obtained for all three assembly strategies.

For the recovered spike-in RNAs, the observed abundances appeared to be highly correlated with the known concentrations in the control solutions (Figure 4B).

Our test data were generated for two groups of juvenile Daphnia: predator-induced and control. Analysis of differential expression with the DESeq2 package revealed 90 upand 64 down-regulated genes in the induced animals, using the RNA-Seq-based reference assembly with a default false discovery rate (FDR) of $10 \%$. The first component in the
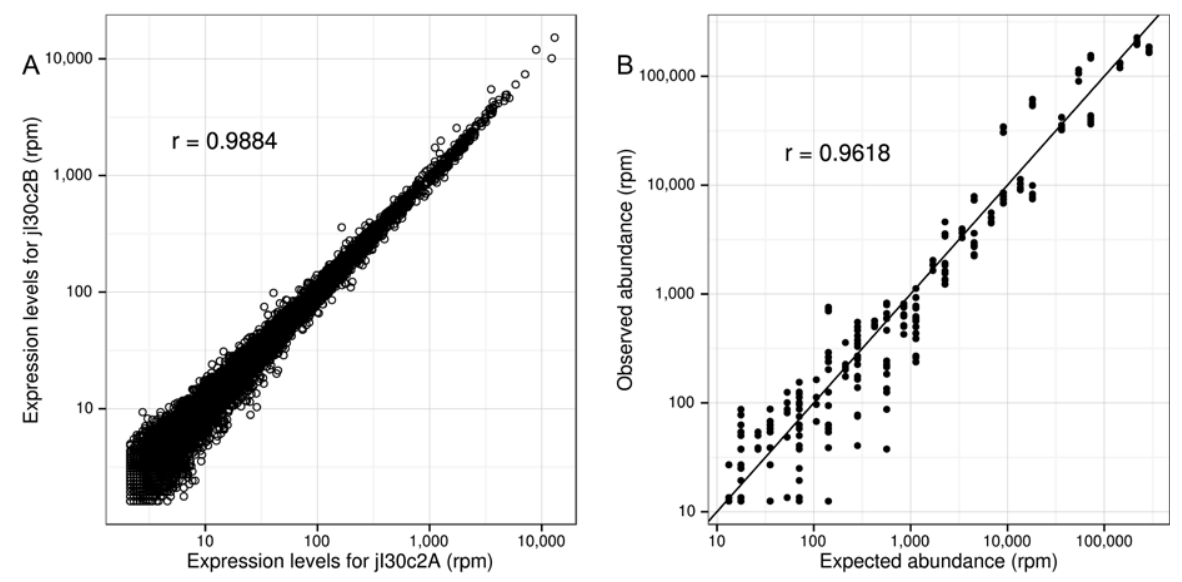

Figure 4. Tag-Seq reproducibility and accuracy. (A) Correlation of the per gene read counts adjusted to reads per million (rpm) between pairs of technical replicates exemplified by sample jl30c2 (raw read counts $<10$ were excluded). (B) Expected and observed abundances of the spike-in control RNAs exemplified by the data for ExFold Mix 2 (four libraries). rpm values lower $<10$ were excluded, leaving 66 control RNAs for the comparison.

principal component analysis allowed us to differentiate the two groups of juveniles, although one of the control clutches (j|30c3) seems to be an outlier since it is similar to the induced juveniles in its gene expression patterns (Supplementary Figure S3). From physiological experiments, it is known that weak spontaneous induction in juvenile Daphnia pulex does take place in laboratory conditions, which might explain the similarity of the jl30c3 sample to the induced animals.

On average 3,524,353 \pm 428,945.4 (mean \pm SD) 50 nucleotide reads per library had exactly 1 match to the transcriptome of the closely related reference species, Daphnia arenata. With stringent quality filtering, we found 49,615 \pm 5739.4 SNP positions covering $9339 \pm 768.0$ loci, with the R9 clone

\section{MYCOPLASMA CONTROL REAGENTS}

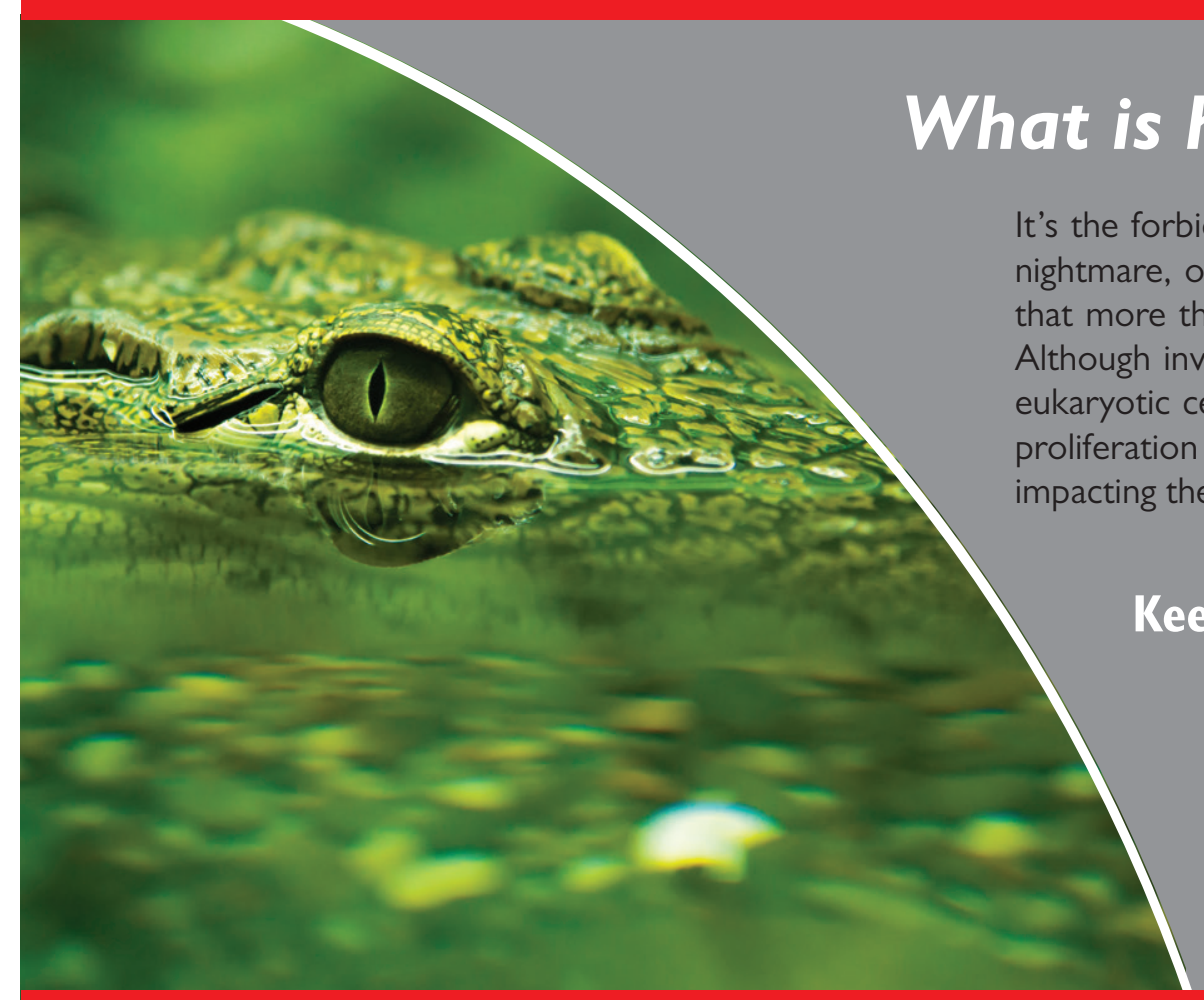

Keep your cells mycoplasma-free:

\section{Detect: PlasmoTest ${ }^{\mathrm{TM}}$}

Eradicate: Plasmocin ${ }^{\mathrm{TM}}$ - Plasmocure ${ }^{\mathrm{TM}}$

Prevent: Normocin ${ }^{\mathrm{TM}}$ - Normocure ${ }^{\mathrm{TM}}$ 
showing confirmed heterozygous states at $8883 \pm 1152.4$ SNPs. Although the values are not directly comparable, a similar number of SNPs was called, for example, in a targeted restriction site-associated DNA sequencing (RAD-Seq) study of intra-specific variation in Daphnia magna (37).

Our approach addresses some of the biases of Tag-Seq while maintaining the technique's advantages for gene expression quantification and SNP discovery. Moreover, the tagging principles described here can also be applied to other types of next-generation sequencing libraries.

\section{Author contributions}

R.T. and F.L. coordinated the project. A.R. designed the protocol, did the laboratory work and bioinformatic analysis, and drafted the manuscript. F.L. and L.W. contributed to the discussion of the protocol. R.T., A.R., L.W., and F.L. designed the experiments.

\section{Acknowledgments}

We thank Uwe John and Nancy Kühne (Alfred Wegener Institute, Helmholz Centre for Polar and Marine Research, Bremerhaven, Germany) for conducting the NextSeq runs and Jan Macher (University of Duisburg and Essen, Germany) for providing the mayfly material.

\section{Competing interests}

The authors declare no competing interests.

\section{References}

1. Mortazavi, A., B.A. Williams, K. McCue, L. Schaeffer, and B. Wold. 2008. Mapping and quantifying mammalian transcriptomes by RNA-Seq. Nat. Methods 5:621-628

2. Wang, Z., M. Gerstein, and M. Snyder. 2009. RNA-Seq: a revolutionary tool for transcriptomics. Nat. Rev. Genet. 10:57-63.

3. Ozsolak, F. and P.M. Milos. 2011. RNA sequencing: advances, challenges and opportunities. Nat. Rev. Genet. 12:87-98.

4. Hansen, K.D., R.A. Irizarry, and Z. Wu. 2012. Removing technical variability in RNA-seq data using conditional quantile normalization. Biostatistics 13:204-216.

5. van Dijk, E.L., Y. Jaszczyszyn, and C. Thermes. 2014. Library preparation methods for next-generation sequencing: tone down the bias. Exp. Cell Res. 322:12-20

6. Asmann, Y.W., E.W. Klee, E.A. Thompson, E.A. Perez, S. Middha, A.L. Oberg, T.M. Therneau, D.I. Smith, et al. 2009. 3' tag digital gene expression profiling of human brain and universal reference RNA using Illumina Genome Analyzer. BMC Genomics 10:531.
7. Hong, L.Z., J. Li, A. Schmidt-Küntzel, W.C. Warren, and G.S. Barsh. 2011. Digital gene expression for non-model organisms. Genome Res. 21:1905-1915.

8. Velculescu, V.E., L. Zhang, B. Vogelstein, and K.W. Kinzler. 1995. Serial analysis of gene expression. Science 270:484-487.

9. Nielsen, K.L., A.L. Høgh, and J. Emmersen. 2006 DeepSAGE-digital transcriptomics with high sensitivity, simple experimental protocol and multiplexing of samples. Nucleic Acids Res. 34:e133.

10. Matsumura, H., K. Yoshida, S. Luo, E. Kimura, T. Fujibe, Z. Albertyn, R.A. Barrero, D.H. Krüger, et al. 2010. High-throughput SuperSAGE for digital gene expression analysis of multiple samples using next generation sequencing. PLoS ONE 5:e12010.

11. Lenz, T.L., C. Eizaguirre, B. Rotter, M. Kalbe, and M. Milinski. 2013. Exploring local immunological adaptation of two stickleback ecotypes by experimental infection and transcriptome-wide digital gene expression analysis. Mol. Ecol. 22:774-786.

12. Armour, C.D., J.C. Castle, R. Chen, T. Babak, P. Loerch, S. Jackson, J.K. Shah, J. Dey, et al. 2009. Digital transcriptome profiling using selective hexamer priming for cDNA synthesis. Nat. Methods 6:647-649.

13. Levin, J.Z., M. Yassour, X. Adiconis, C. Nusbaum, D.A. Thompson, N. Friedman, A. Gnirke, and A. Regev. 2010. Comprehensive comparative analysis of strand-specific RNA sequencing methods. Nat. Methods 7:709-715.

14. Hashimshony, T., F. Wagner, N. Sher, and I. Yanai. 2012. CEL-Seq: single-cell RNA-Seq by multiplexed linear amplification. Cell Rep. 2:666-673.

15. Kahl, G., C. Molina, B. Rotter, R. Jüngling, A. Frank, N. Krezdorn, K. Hoffmeier, and P. Winter. 2012. Reduced representation sequencing of plant stress transcriptomes. J. Plant Biochem. Biotechnol. 21(Suppl. 1):119-127.

16. Zawada, A.M., K.S. Rogacev, S. Müller, B. Rotter, P. Winter, D. Fliser, and G.H. Heine. 2014. Massive analysis of cDNA ends (MACE) and miRNA expression profiling identifies proatherogenic pathways in chronic kidney disease. Epigenetics 9:161.

17. Zajac, B.K., J. Amendt, R. Horres, M. Verhoff, and R. Zehner. 2015. De novo transcriptome analysis and highly sensitive digital gene expression profiling of Calliphora vicina (Diptera: Calliphoridae) pupae using MACE (Massive Analysis of cDNA Ends). Forensic Sci. Int. Genet. 15:137-146.

18. Brenner, S., M. Johnson, J. Bridgham, G. Golda, D.H. Lloyd, D. Johnson, S. Luo, S. McCurdy, et al. 2000. Gene expression analysis by massively parallel signature sequencing (MPSS) on microbead arrays. Nat. Biotechnol. 18:630-634.

19. Kim, J.B., G.J. Porreca, L. Song, S.C. Greenway, J.M. Gorham, G.M. Church, C.E. Seidman, and J. Seidman. 2007. Polony multiplex analysis of gene expression (PMAGE) in mouse hypertrophic cardiomyopathy. Science 316:1481-1484.

20. Ozsolak, F., A. Goren, M. Gymrek, M. Guttman, A. Regev, B.E. Bernstein, and P.M. Milos. 2010. Digital transcriptome profiling from attomole-level RNA samples. Genome Res. 20:519-525.

21. Casbon, J.A., R.J. Osborne, S. Brenner, and C.P. Lichtenstein. 2011. A method for counting PCR template molecules with application to next-generation sequencing. Nucleic Acids Res. 39:e81.

22. Kivioja, T., A. Vähärautio, K. Karlsson, M. Bonke, M. Enge, S. Linnarsson, and J. Taipale. 2012. Counting absolute numbers of molecules using unique molecular identifiers. Nat. Methods 9:72-74.
23. Schweyen, H., A. Rozenberg, and F. Leese. 2014. Detection and removal of PCR duplicates in population genomic ddRAD studies by addition of a degenerate base region (DBR) in sequencing adapters. Biol. Bull. 227:146-160.

24. Tollrian, R. 1993. Neckteeth formation in Daphnia pulex as an example of continuous phenotypic plasticity: morphological effects of Chaoborus kairomone concentration and their quantification. J. Plankton Res. 15:1309-1318.

25. Repka, S., M. Walls, and M. Ketola. 1995. Neck spine protects Daphnia pulex from predation by Chaoborus, but individuals with longer tail spine are at a greater risk. J. Plankton Res. 17:393-403.

26. Tollrian, R. 1995. Predator-induced morphological defenses: costs, life history shifts, and maternal effects in Daphnia pulex. Ecology 76:1691-1705.

27. Bolger, A.M., M. Lohse, and B. Usadel. 2014. Trimmomatic: a flexible trimmer for Illumina sequence data. Bioinformatics 30:2114-2120.

28. Langmead, B. and S.L. Salzberg. 2012. Fast gapped-read alignment with Bowtie 2. Nat. Methods 9:357-359.

29. Love, M.I., W. Huber, and S. Anders. 2014 Moderated estimation of fold change and dispersion for RNA-seq data with DESeq2. Genome Biol. 15:550

30. Li, H., B. Handsaker, A. Wysoker, T. Fennell, J. Ruan, N. Homer, G. Marth, G. Abecasis, and R. Durbin. 2009. The sequence alignment/map format and SAMtools. Bioinformatics 25:2078-2079.

31. Grabherr, M.G., B.J. Haas, M. Yassour, J.Z. Levin, D.A. Thompson, I. Amit, X. Adiconis, L. Fan, et al. 2011. Full-length transcriptome assembly from RNA-Seq data without a reference genome. Nat. Biotechnol. 29:644-652.

32. Lee, C., R.A. Harris, J.K. Wall, R.D. Mayfield, and C.O. Wilke. 2013. RNasell and T4 Polynucleotide Kinase sequence biases and solutions during RNA-seq library construction. Biol. Direct 8:16.

33. Turunen, J.J., L.V. Pavlova, M. Hengesbach, M. Helm, S. Müller, R.K. Hartmann, and M.J. Frilander. 2014. RNA ligation, p. 45-88. In R.K. Hartmann, A. Bindereif, A. Schön, and E. Westhof (Eds.), Handbook of RNA Biochemistry, 2nd Ed. Wiley-VCH Verlag, Weinheim, Germany

34. Jayaprakash, A.D., O. Jabado, B.D. Brown, and R. Sachidanandam. 2011. Identification and remediation of biases in the activity of RNA ligases in small-RNA deep sequencing. Nucleic Acids Res. 39:e141.

35. Romaniuk, E., L.W. McLaughlin, T. Neilson, and P.J. Romaniuk. 1982. The effect of acceptor oligoribonucleotide sequence on the T4 RNA ligase reaction. Eur. J. Biochem. 125:639-643.

36. Griebel, T., B. Zacher, P. Ribeca, E. Raineri, V. Lacroix, R. Guigó, and M. Sammeth. 2012. Modelling and simulating generic RNA-Seq experiments with the flux simulator. Nucleic Acids Res. 40:10073-10083

37. Fields, P.D., C. Reisser, M. Dukic' , C.R. Haag, and D. Ebert. 2015. Genes mirror geography in Daphnia magna. Mol. Ecol. 24:4521-4536.

Received 14 September 2015; accepted 12 April 2016.

Address correspondence to Andrey Rozenberg Department of Animal Ecology, Evolution and Biodiversity, Ruhr University Bochum, Universitaetsstrasse 150, D-44801 Bochum, Germany. E-mail: andrey.rozenberg@rub.de, jaera@yandex.com

\section{To purchase reprints of this article, contact:} biotechniques@fosterprinting.com 